\title{
Valoración sociocultural de servicios ecosistémicos a nivel local
}

\section{Sociocultural assessment of ecosystem services at local level}

\author{
Norvy Yaqueline Náñez Martínez ${ }^{1}$ \\ Lilia Graciela Bustamante Vidal², \\ Ivonne Andrea Narváez- Zambrano ${ }^{3}$, \\ Daniel Andrés Fériz-García ${ }^{4}$.
}

'Ecóloga, Semillero de investigación en Gestión y Legislación ambiental. Fundación Universitaria de Popayán.

Dirección: Corregimiento del estrecho, Patía. E-mail:yaque508@hotmail.com

${ }^{2}$ Estudiante programa Ecología, Semillero de investigación en Gestión y Legislación ambiental. Fundación Universitaria de Popayán.

${ }^{3}$ Magister en Ciencias Forestales, Ecóloga, investigadora Grupo de Investigación UNIET, Programa de Ecología, Fundación Universitaria de Popayán.

E-mail: ivonne.narvaez@docente.fup.edu.co Dirección: Sede Claustro San Fosé Calle 5 No. 8-58

${ }^{4}$ Magíster en Recursos Hidrobiológicos, Biólogo, investigador Grupo de Investigación UNIET, Programa de Ecología, Fundación Universitaria de Popayán.

\section{Resumen}

La valoración sociocultural de los Servicios Ecosistémicos (SE) es un abordaje que explora la percepción que diferentes actores interesados tienen sobre los SE, por lo tanto, permite establecer las relaciones de pertenencia y apropiación existentes entre las comunidades y su territorio. Esta investigación describe cómo los SE que brinda la vereda Pueblillo -Popayán, (Cauca,
Historia del artículo

Fecha de recepción:

27-08-2021

Fecha de aceptación:

09-11-2021

DOI: https://doi.

org/10.47374/

novcol.2021.v16.2003 
Colombia) son identificados y valorados por organizaciones sociales, residentes de Pueblillo, actores institucionales y academia. Para la recolección de datos se implementaron técnicas no probabilísticas, muestreo intencional y bola de nieve. Adicionalmente, se diseñaron cuestionarios estructurados, semiestructurados y se realizaron entrevistas cara a cara. No hubo diferencia significativa entre los tipos de SE identificados por los actores interesados, sin embargo, el servicio de aprovisionamiento fue mayormente identificado $(50,1 \%)$, seguido por el servicio de regulación (28.5\%) y el servicio cultural $(28,5 \%)$. Los actores interesados identificaron quince subcategorías de servicios de aprovisionamiento, once subcategorías de servicios de regulación y nueve subcategorías de servicios culturales. Se evidenció que los actores con alta dependencia de los SE, dan mayor importancia a los servicios de aprovisionamiento mientras que los actores con alta influencia sobre los SE, se la dan a los de regulación.

Por su parte, los actores de la vereda Pueblillo no perciben el alto potencial que ofrecen los servicios ecosistémicos culturales para la dinamización de estrategias de innovación social que aporten al desarrollo local desde el aprovechamiento cultural del territorio, por lo que se requiere implementar un proceso de valoración económica ambiental y de capacitación en modelos de gestión campesina multifuncional, turismo cultural y de aventura.
Palabras claves: Valoración socio cultural, actores interesados, servicios ecosistémicos.

\section{Abstract}

The sociocultural valuation of ecosystem services (ES) is an approach that explores the perception that different stakeholders have about ES, therefore, it allows establishing the existing relationships of belonging and appropriation between communities and their territory. This research describes how the ES provided by the Pueblillo village -Popayán, (Cauca, Colombia) are identified and valued by social organizations, Pueblillo residents, institutional actors and academia. Nonprobabilistic techniques, Purposive sampling and snowball were used for data collection. Additionally, structured and semi-structured questionnaires were designed and face-to-face interviews were conducted. There was not significant difference between the types of ES identified by the stakeholders, however, the provisioning service was mostly identified (50.1\%), followed by the regulation service $(28.5 \%)$ and the cultural service (28.5\%). \%). Stakeholders identified 15 subcategories of provisioning services, 11 subcategories of regulation services, and 9 subcategories of cultural services. It was evidenced that the actors with high dependence on the ES, give greater importance to the provisioning services, while the actors with high influence on the ES, give it to the regulators. The actors of the village of Pueblillo do not perceive the high potential offered by cultural ecosystem services for the dynamization of 
social innovation strategies that contribute to local development from the cultural use of the territory, so it is necessary to implement a process of environmental economic valuation and training in peasant multifunctional management models, cultural and adventure tourism.

Keywords: Socio-cultural assessment, stakeholders, ecosystem services.

\section{Introducción}

Los servicios ecosistémicos (SE), se definen como los componentes y procesos de los ecosistemas que aumentan el bienestar de la humanidad cuando son consumidos o disfrutados (Quijas et al. 2010). Estos están sujetos a múltiples valores que se abordan desde los enfoques biofísico, monetario y sociocultural (de Groot et al., 2010). El primero, caracteriza los componentes del ecosistema y los procesos ecológicos necesarios para su suministro (Balvanera et al., 2011); el segundo, busca determinar su valor económico (Vihervaara et al., 2010) y justificar la inversión en políticas de conservación (La Notte et al, 2013); y el tercero, estima los valores, actitudes y significados que acentúan la demanda de SE (Bullock, Joyce y Collier, 2018). Tal valoración integral es usada en la creación de marcos conceptuales para sostener ecosistemas sanos y diversos que aseguren el bienestar de las sociedades (Balvanera et al, 2013). Sin embargo, las valoraciones biofísicas y monetarias a menudo prevalecen sobre las socioculturales (Nieto-Romero et al, 2014), tanto en la investigación como en la integración de políticas para la gestión territorial y el manejo del capital natural (Chang et al., 2012). La disparidad en la evaluación de los SE, compromete la comprensión sobre interacciones entre personas y ecosistemas, que, en última instancia, determinan el uso, manejo, toma de decisiones y capacidad de los sistemas terrestres para proporcionar SE (Balvanera et al, 2015). Concretamente, se limita el reconocimiento de valores sociales y beneficios de la naturaleza que por no ser monetarios son menos tangibles (Malinauskaite et al., 2021).

Por todo lo sostenido, la valoración sociocultural representa un abordaje necesario para capturar información sobre la percepción de importancia que diferentes actores interesados tienen sobre los servicios ecosistémicos (Zograftos y Kumar 2010; Arianda et al., 2014). En efecto, considerar a los actores interesados, permite conocer las diferencias en las motivaciones y grados de influencia (Reeds et al, 2017), puesto que sus percepciones se relacionan con los contextos sociales, los modos de vida, las tradiciones culturales, las necesidades individuales, los intereses profesionales, el acceso a los SE y el tipo de conocimiento sobre ellos (Martín-López et al., 2012).

Además, los ejercicios de valoración sociocultural se benefician de la inclusión de actores interesados locales y diferentes, porque aportan mayor cantidad de conocimientos locales, técnicos y experienciales; permiten resolver conflictos con mayor éxito (Tëngo et al., 2014) y abordan las desigualdades 
presentes en diferentes contextos sociales y culturales, puesto que, quienes presentan una alta dependencia de los SE, no necesariamente logran participar de su gestión (Vallet et al, 2019). En escalas locales de valoración sociocultural, las decisiones que afectan directamente la gestión de los ecosistemas, se toman en su mayoría a nivel local y están influenciadas por los valores biofísicos y socioculturales atribuidos a los servicios (Kandel et al, 2018), por lo tanto, el uso de este tipo de valoración permite procesos más participativos, al considerar en la planeación y gestión territorial, las diferentes percepciones de los actores interesados (Maynard et al. 2010; Aryal, Ojha y Maraseni, 2021), más aún, cuando se considera que, las realidades socioculturales específicas de las comunidades que tienen poca influencia en la toma de decisiones, generalmente son ignoradas en la gestión ambiental (García-Nieto et al., 2015).

Entre los actores interesados existen motivaciones de tipo pragmático o normativo (ej. proteger el medio ambiente o generar confianza en las instituciones respectivamente), que están vinculadas al logro de resultados de interés particular, según el ámbito en el que participan (de Vente et al. 2016). Adicionalmente, los actores interesados intercambian conocimiento e información, tanto uni como bidireccionalmente, por lo tanto, se originan modos de participación más consultiva o más deliberativa (Reed et al, 2017). Este contexto, también involucra la capacidad de los actores interesados para influenciar una decisión o ser influenciados por ella. En resumen, intereses, motivaciones, grados de influencia y modos de comunicación, caracterizan las formas de participación activa o pasiva de los actores interesados.

Con respecto a la percepción sobre los SE de los actores interesados, estudios realizados en varias partes del mundo, incluyendo América Latina, muestran que diferentes SE son considerados como importantes por diversos grupos de actores interesados locales, que tienen diferentes intereses sociales (Tëngo et al., 2014; García-Nieto et al., 2015). Las preferencias por algún tipo de SE se deben principalmente a los beneficios percibidos por el servicio que se suministra al grupo de actores interesados (Yameogo, 2017) y que en varios contextos satisfacen necesidades básicas de subsistencia, como la producción de alimentos (Vilardy et al., 2012) o porque la dependencia local de los SE es alta (Turner el al, 2012), por lo tanto, estos servicios son catalogados como muy importantes (Alpuche et al., 2019).

Trabajos de valoración sociocultural a escala local muestran que pobladores locales otorgaron mayor valoración a los servicios de aprovisionamiento que a los servicios de regulación (Mensah et al, 2017); actores con diferentes intereses económicos también perciben como importantes los servicios de aprovisionamiento (Iniguez-Gallardo et al, 2018). Adicionalmente, las preferencias de los actores interesados están relacionadas con la influencia que estos tienen sobre los SE, como ocurre con administradores 
ambientales, gestores e investigadores, quienes otorgan un peso importante a los servicios de regulación (Iniesta-Arianda et al., 2014; Aguado et al., 2018). Esta inclinación es explicada por el conocimiento experiencial y técnico de los actores interesados (GarcíaNieto et al., 2015).

Con base en estas evidencias, se plantea como hipótesis que, las percepciones, valoraciones y participación de los actores interesados de la vereda Pueblillo, estarán direccionadas hacia servicios ecosistémicos asociados con los modos de vida, puesto que, tanto quienes se benefician de los servicios, como quienes los gestionan, realizan elecciones intencionales que resultan de los intereses y motivaciones sobre el territorio y del grado de influencia o dependencia que se tiene sobre los SE, llevando a la preferencia por los beneficios locales (Vallet et al, 2019), que determinan la demanda de uno u otro tipo de servicio. Además, en entornos socialmente desiguales, los actores interesados asumen roles fuertemente marcados que limitan la participación equitativa en los procesos de toma de decisiones sobre SE (FelipeLucia et al, 2015) que les permita ampliar las percepciones hacia servicios menos tangibles para ellos. La comprensión de las preferencias y valoraciones de los SE hechas por los actores interesados, es un insumo importante para la gestión ambiental y el ordenamiento territorial, ya que, en la zona se han disturbado áreas ambientales estratégicas para el suministro de servicios ecosistémicos, como el mantenimiento de la calidad del aire, el control de la erosión, la purificación del agua, la belleza escénica y la recreación.

Para verificar la hipótesis, se plantea un estudio de caso que caracteriza las percepciones de los actores interesados sobre los servicios ecosistémicos de la vereda Pueblillo, municipio de Popayán, departamento del Cauca (Colombia). Se enfatiza en identificar los servicios ecosistémicos y en determinar la importancia que se les otorga. Los resultados permiten informar a los tomadores de decisiones y al público en general sobre los valores socioculturales de los SE para su incorporación en la gestión sostenible del capital natural de la vereda y en la estructuración de estrategias para la conservación, que permitan mantener los medios de vida periurbanos.

\section{Materiales y Métodos}

\section{Área de estudio}

La vereda Pueblillo está situada en la cuenca media del río Molino al Nororiente de la ciudad de Popayán, departamento del Cauca (Colombia), a $2{ }^{\circ} 26^{\prime} \mathrm{N}$ y $76^{\circ} 35^{\prime} \mathrm{O}$. Su altitud es de $1700 \mathrm{msnm}$, con temperatura media anual de $19^{\circ} \mathrm{C}$, (Ramírez-Chaves, Pérez y Mosquera, 2008) y una extensión de 20,6 hectáreas (Gómez, 2012). Pertenece a la zona de vida bosque húmedo Montano Bajo (bhMB), según Holdridge (1982). Su vegetación sucesional se caracteriza por la presencia de pastos enmalezados, vegetación secundaria con estratos herbáceos y arbustivos y bosque denso con menos del $80 \%$ de cobertura, 
debido al consumo de leña y actividades agrícolas en áreas periféricas. El uso del suelo se distribuye principalmente entre cultivos de autoconsumo de: maíz, hortalizas, caña, plátano y agricultura semi-intensiva de café; extracción de arcillas, gravas y recebos (POT, 2002; SGC, 2015) (Figura 1).

\section{Recolección de datos}

Se utilizó un enfoque de tipo cualitativo para comprender las interpretaciones que cada actor interesado tiene sobre los servicios ecosistémicos. Se adoptaron dos técnicas de recolección de datos no

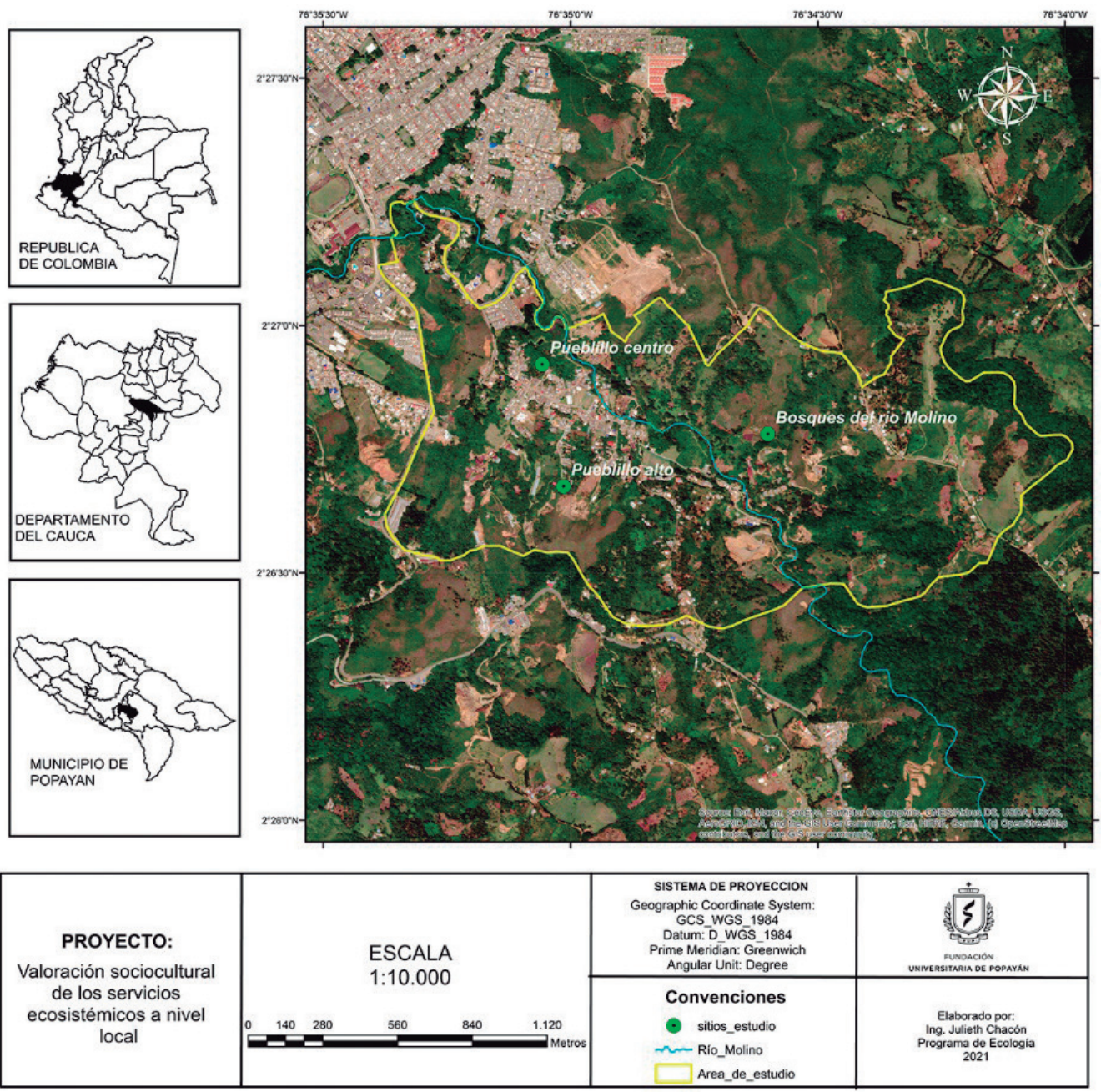

Figura 1: Mapa de los sitios de estudio en la vereda Pueblillo que corresponden a delimitaciones no oficiales realizadas por la comunidad. 
probabilísticas: muestreo intencional, con el que se identifican y seleccionan individuos o grupos de individuos informados o con experiencia en el tópico de interés (Cresswell y Plano Clark, 2011); bola de nieve, que permite a cada entrevistado referenciar a otro actor, hasta llegar al punto de saturación (Reed, 2009), lo cual resulta pertinente cuando no existe un marco de muestreo para la zona (Anheier y Katz, 2004).

\section{Selección de participantes}

Mediante el muestreo intencional se realizó una revisión documental de la zona de estudio para identificar deliberadamente actores locales según su interés particular en el uso o gestión de los servicios ecosistémicos. Estos fueron clasificados en función del nivel de influencia o dependencia frente a los SE (Figura 2), mediante la adaptación de

\section{NIVEL DE INFLUENCIA}

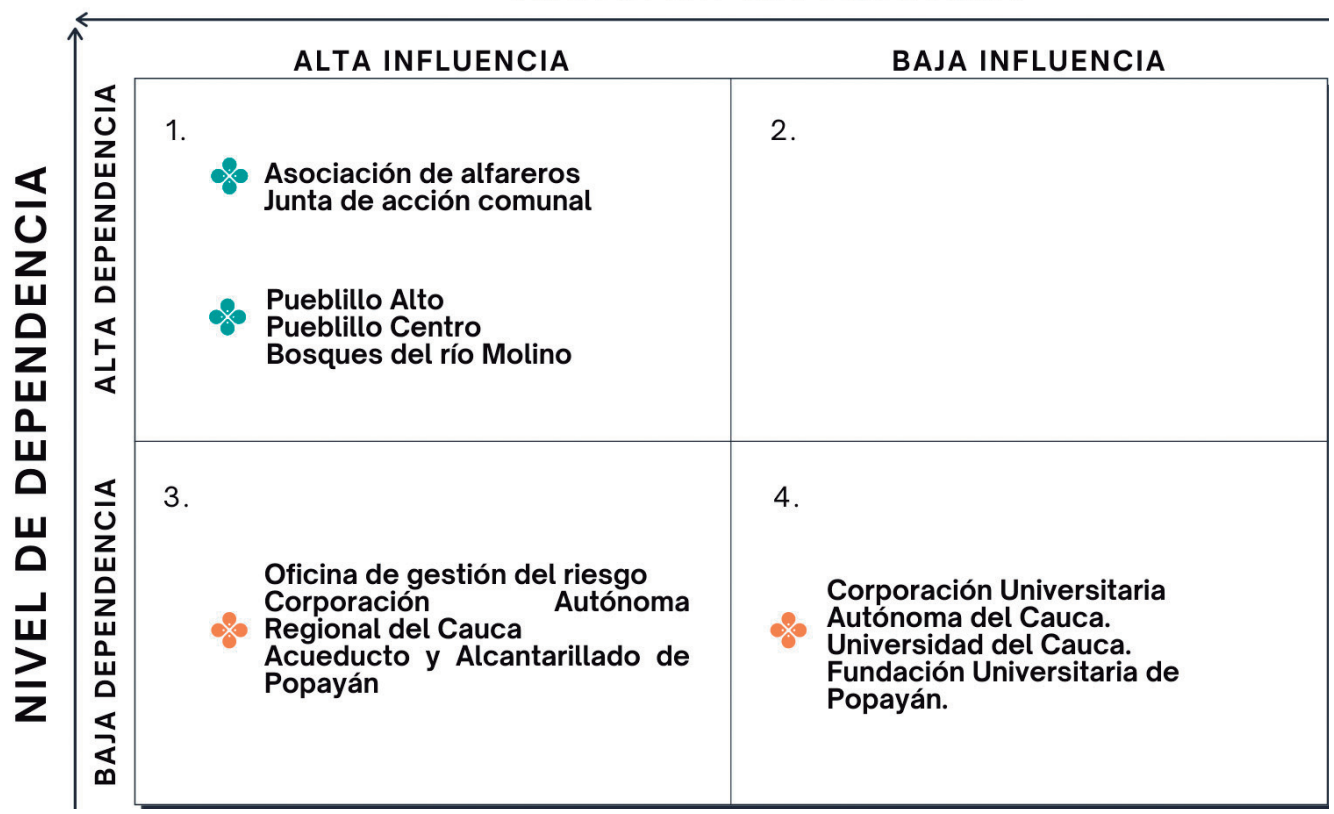

Figura 2. Matriz de dependencia-influencia para priorizar los actores interesados (Adaptado de, de Groot et al, 2006). 1. Actores interesados expuestos a una importante pérdida o ganancia, según las medidas de gestión adoptadas en el socio-ecosistema. 2. Actores interesados expuestos a una importante pérdida o ganancia, según las medidas de gestión adoptadas en el socio-ecosistema, sin embargo, sus acciones o decisiones no afectan al flujo de servicios. 3. Actores interesados cuyas acciones o decisiones pueden afectar la capacidad del ecosistema para suministrar servicios, pero que no se ven afectados

(positiva o negativamente) por el cambio en el flujo de servicios. 4. Actores interesados que no están sometidos a una importante pérdida o ganancia en función de las medidas de gestión adoptadas en el socio-ecosistema y cuyas acciones no afectan al flujo de servicios. 
la matriz propuesta por De Groot et al, (2006). El nivel de influencia se refiere al grado de control, acceso o manejo que el grupo de actores interesados tiene sobre los servicios de los ecosistemas; el nivel de dependencia hace referencia al efecto que tienen los servicios de los ecosistemas sobre el bienestar del grupo de actores. Los actores primarios son aquellos cuyo bienestar humano depende del suministro de servicios y los actores secundarios son aquellos con elevada influencia sobre el suministro de servicios. Los actores interesados de cada categoría fueron incorporados hasta llegar al punto de saturación, a través de la técnica "bola de nieve" (Instituto Humboldt, 2014).
Identificación y valoración

de los servicios ecosistémicos

Se utilizaron cuestionarios estructurados para la fase piloto y semiestructurados para la fase definitiva. La fase piloto proporcionó información sociodemográfica de edad, género, nivel de educación y actividad económica, además, información sobre los servicios ecosistémicos que los actores interesados identificaron en la zona de estudio. Los actores interesados respondieron un cuestionario en línea sin restricciones de contraseña o registro, a través de la plataforma Survey Monkey en el que podían elegir entre una lista de SE organizados según la tipología establecida

Tabla 1. Clasificación de los servicios ecosistémicos según la VIBSE

\begin{tabular}{|c|c|c|}
\hline Tipo SE & Definición & Ejemplos - subcategorias \\
\hline $\begin{array}{c}\text { Servicios de } \\
\text { aprovisionamiento }\end{array}$ & $\begin{array}{l}\text { Productos } \\
\text { obtenidos de los } \\
\text { ecosistemas. }\end{array}$ & $\begin{array}{c}\text { Agricultura, ganadería, pesca, acuicultura, } \\
\text { alimentos silvestres, agua para consumo humano, } \\
\text { agua para consumo agrícola, madera, forraje } \\
\text { y pasto, especies ornamentales, suministro de } \\
\text { arcilla, materiales de río, especies autóctonas de } \\
\text { plantas, especies autóctonas de animales, plantas } \\
\text { aromáticas. }\end{array}$ \\
\hline $\begin{array}{l}\text { Servicios de } \\
\text { regulación }\end{array}$ & $\begin{array}{l}\text { Procesos de } \\
\text { autorregulación } \\
\text { de los ecosistemas } \\
\text { que ayudan a } \\
\text { reducir impactos } \\
\text { locales y globales. }\end{array}$ & $\begin{array}{l}\text { Clima local y regional, captura y almacenamiento } \\
\text { de } \mathrm{CO}_{2} \text {, reducción de escorrentía, purificación } \\
\text { del agua, purificación del aire, control de la } \\
\text { erosión, retención de humedad, control biológico, } \\
\text { polinización, hábitat de especies singulares. }\end{array}$ \\
\hline Servicios culturales & $\begin{array}{l}\text { Beneficios no } \\
\text { materiales } \\
\text { obtenidos de los } \\
\text { ecosistemas }\end{array}$ & $\begin{array}{l}\text { Educación ambiental, centros de interpretación, } \\
\text { creencias y costumbres, modos tradicionales, } \\
\text { admiración del paisaje, avistamiento de aves, } \\
\text { balnearios, senderismo, ciclismo. }\end{array}$ \\
\hline
\end{tabular}

Fuente: Modificado de Valoración integral de la biodiversidad y los servicios ecosistémicos (2014) 
por la VIBSE (Tabla 1). La tipología incluyó una explicación sobre el significado de cada servicio ecosistémico (aprovisionamiento, regulación y cultural), para favorecer una mejor comprensión de los participantes. Esta fase preliminar permitió mejorar el diseño y la comprensión del cuestionario definitivo.

En la fase definitiva se realizaron entrevistas cara a cara para las que se usó un cuestionario ajustado. Se explicó el objetivo y la confidencialidad de las respuestas, además, se solicitó autorización para realizar grabaciones y tomar notas. Para valorar la importancia otorgada a cada SE, se utilizó en la primera parte del cuestionario la escala de Likert con 5 valores, donde 1 es no importante, 2 poco importante, 3 moderadamente importante, 4 importante y 5 extremadamente importante. La segunda parte del cuestionario utilizó preguntas abiertas para conocer el tipo de suministro específico para las categorías de servicios. Las entrevistas se desarrollaron en los meses de abril y junio de 2021.

\section{Análisis de datos}

El procesamiento y análisis de la información se realizó con el programa estadístico SPSS 25.0. (IBM Corp, 2017). Para las variables actores interesados, edad, género, nivel de educación, actividad económica y tipos de servicios ecosistémicos, los datos se organizaron mediante tablas de frecuencia; adicionalmente, se estableció la relación entre las variables independientes, actores interesados, edad, género, nivel de educación, actividad económica y tipos de servicios ecosistémicos versus la variable de respuesta y nivel de importancia mediante tablas de contingencia. Para determinar el grado de asociación o dependencia entre las variables categóricas analizadas se utilizó la prueba estadística Chí-cuadrado de Pearson $\left(\mathrm{X}^{2}\right)$ para tablas de contingencia $\mathrm{n} \times \mathrm{n}$.

\section{Resultados y discusión}

\section{Caracterización de los participantes \\ Tipo de actores interesados}

Esta priorización permitió determinar la presencia de actores interesados clasificados como primarios y secundarios (Tabla 2):

a) Los actores primarios fueron aquellos con una alta dependencia del suministro de SE, asociada a la satisfacción de necesidades básicas, como el consumo de alimentos y de agua potable y el acceso a fuentes de ingresos económicos, principalmente la agricultura y la extracción de arcilla y materiales de río. Los actores tienen una influencia limitada en la gestión de los servicios porque no están suficientemente involucrados en espacios de organización comunitaria, tanto para una mejor participación en la resolución de problemas sociales, culturales, económicos, políticos y productivos que inciden en las dinámicas ambientales de su territorio, como para influir en la toma de decisiones sobre el uso de los recursos naturales y la gestión ambiental. Esta realidad se refleja en la división del territorio que identificó la comunidad en tres sectores (Figura 1), cada uno con junta de acción comunal 
que no está reconocida antes los entes gubernamentales. Además, los residentes manifestaron que, existen desavenencias entre los tres sectores, poco interés en participar de los procesos desarrollados por las juntas y desarticulación entre ellas. Los conflictos entre las partes interesadas a menudo están relacionados con el tipo de participación que lleva a una variedad de resultados, positivos e indeseados, $\mathrm{y}$ aunque en la literatura actual no está claro aún por qué los diferentes procesos de participación conducen a resultados tan diferentes, existen modelos como "la rueda de la participación" (Reed, 2017), que pueden ayudar a comprender de forma más rigurosa y útil, el tipo de participación que se da entre los actores interesados de Pueblillo.

Concretamente, las dinámicas de participación manifestadas por los actores interesados se pueden asignar al tipo de participación denominado "deliberación y/o coproducción de arriba hacia abajo" (Top-down deliberation and/or coproduction), en el que la participación es iniciada y dirigida de arriba hacia abajo por actores institucionales y organizaciones sociales (Tabla 2) con poder para la toma de decisiones; aquí los residentes son involucrados en deliberaciones bidireccionales, pero la decisión final, no siempre es tomada conjuntamente y queda a cargo de actores interesados, como la Oficina de Gestión del Riesgo, la Corporación Autónoma Regional del Cauca (CRC), el Acueducto y Alcantarillado de Popayán, la Asociación de Alfareros de Pueblillo y las Juntas de acción comunal. Este hecho puede provocar que las realidades socioculturales específicas de las comunidades con poca influencia en la toma de decisiones sean ignoradas en la planificación y toma de decisiones de gestión ambiental (Maguire, Potts y Flectcher, 2012). Para Durham, et al (2019) el uso del conocimiento local de las partes interesadas que dependen directamente de los SE puede ayudar a identificar soluciones aceptables que se ajusten mejor a las condiciones locales.

b) Actores secundarios institucionales. Estos presentaron una alta influencia sobre los SE, puesto que se encargan de regular el uso de los recursos naturales en la vereda y pueden influir en la toma de decisiones sobre políticas de manejo de los recursos (Benítez et al., 2013) y a menudo, definen el tipo de información que se utiliza en la toma de decisiones (Ruhl, 2016). También se encontró que la mayoría de estos actores son residentes de la zona de estudio, de ahí que se configure un nivel diferente de dependencia de los SE, porque, si bien no centran la satisfacción de necesidades económicas en la extracción de materias primas, sí se benefician de la producción de alimentos de la zona, porque manifiestan que son más económicos y son producidos por sus vecinos. Varios estudios resaltan el papel del contexto local en los tipos de participación de los actores interesados (Ghai y Vivian, 2014; COM, 2017). Para el caso de la vereda Pueblillo, esto representa una oportunidad para fortalecer la participación de abajo hacia arriba (Bottom-up deliberation and/ or coproduction) (Reeds et al, 2017), donde esta sea iniciada y dirigida por los residentes de Pueblillo y las decisiones se tomen 
basándose en el conocimiento adquirido a través deliberaciones bidireccionales de los actores interesados. El fortalecimiento de las capacidades de base favorece el empoderamiento de la comunidad local y permite que alrededor del uso común de recursos se amplíe la gama de puntos de vista e innovaciones, situación diferente a los procesos tradicionales de arriba hacia abajo (Top-down one-way communication and/or consultation) (Primmer et al., 2015; Reeds, et al., 2017).

Con respecto a la academia, su influencia es limitada en las decisiones y políticas de manejo de los recursos, ya que los resultados de investigaciones no son integrados a los instrumentos de planificación del territorio. Esto sugiere que entre investigadores y demás actores interesados no siempre se manejan los mismos intereses y expectativas sobre los procedimientos, resultados y estándares de calidad de las investigaciones que las lleven a ser consideradas como preponderantes para la toma de decisiones sobre la gestión ambiental del territorio. Se reconoce en la literatura que la transferencia de conocimiento científico a los espacios de formulación de políticas y toma de decisiones no ocurre linealmente y no garantiza de forma exclusiva una mejor toma de decisiones (Saarikoski, 2018). Es ahí donde se crean brechas entre la academia, residentes locales y actores institucionales, que obedecen generalmente a la insuficiencia de métodos que sean fáciles de entender, transferir, escalar, evaluar y que sean rentables (Olander et al., 2017). Los desafíos para las instituciones y la academia, plantean la necesidad de realizar trabajos interdisciplinarios que adecúen las evidencias técnicas y los argumentos científicos sobre servicios ecosistémicos al contexto de la práctica de la gestión ambiental (Hauck et al., 2016).

Además, se hace necesario comprender los tipos de participación que se configuran en el territorio, reconociendo el contexto local, el diseño de los procesos de participación, la mediación a través de enfoques cooperativos que demuestran mejores resultados en la relación entre los actores interesados, el aprendizaje social, la implementación de la legislación ambiental (Reeds, 2017) y la democracia que legitima los tipos de participación al incluir las contribuciones, los valores y las opiniones de varios actores interesados (Hauck et al, 2016; Reeds, 2017).

\section{Características sociodemográficas}

de los actores interesados

Edad y genero de los actores interesados

La franja etaria de los actores interesados se encuentra entre 20 y 77 años, con una media de 45 años. Esto sugiere que, al encontrarse en un periodo potencialmente productivo desde el punto de vista laboral, podrían acceder a mayores posibilidades de crecimiento económico y mejores niveles de bienestar (CEPAL, 2008). En concordancia, se evidenció mayor porcentaje de ocupaciones $(80 \%)$, que les permiten tener un ingreso económico ya sea como empleados o trabajadores independientes, estos últimos, en 
Tabla 2: Actores interesados en la vereda Pueblillo. Actores primarios: su bienestar depende del suministro de servicios; actores secundarios: elevada influencia sobre el suministro de servicios.

\begin{tabular}{|c|c|c|c|c|}
\hline $\begin{array}{c}\text { Nivel de } \\
\text { influencia } \\
\text { y dependencia }\end{array}$ & Tipo de actor & Denominación & $\begin{array}{l}\text { No. de } \\
\text { respuestas }\end{array}$ & Porcentaje \\
\hline \multirow{5}{*}{$\begin{array}{l}\text { Actores } \\
\text { primarios }\end{array}$} & \multirow{2}{*}{$\begin{array}{l}\text { Organizaciones } \\
\text { sociales }\end{array}$} & $\begin{array}{l}\text { Asociación de Alfareros de } \\
\text { Pueblillo }\end{array}$ & 4 & $23,14 \%$ \\
\hline & & Junta de acción comunal. & 3 & \\
\hline & \multirow{3}{*}{$\begin{array}{l}\text { Residentes de } \\
\text { Pueblillo }\end{array}$} & Pueblillo Alto & 5 & \\
\hline & & Pueblillo Centro & 7 & $37,03 \%$ \\
\hline & & Bosques del río Molino & 10 & \\
\hline \multirow{6}{*}{$\begin{array}{c}\text { Actores } \\
\text { secundarios }\end{array}$} & \multirow{3}{*}{$\begin{array}{c}\text { Actores } \\
\text { institucionales }\end{array}$} & Oficina de gestión del riesgo & 1 & \\
\hline & & $\begin{array}{l}\text { Corporación Autónoma } \\
\text { Regional del Cauca }\end{array}$ & 2 & $25,67 \%$ \\
\hline & & $\begin{array}{c}\text { Acueducto y Alcantarillado de } \\
\text { Popayán }\end{array}$ & 1 & \\
\hline & \multirow{3}{*}{ Academia } & $\begin{array}{c}\text { Corporación Universitaria } \\
\text { Autónoma del Cauca }\end{array}$ & 1 & \\
\hline & & Universidad del Cauca & 1 & $14,17 \%$ \\
\hline & & $\begin{array}{l}\text { Fundación Universitaria de } \\
\text { Popayán }\end{array}$ & 2 & \\
\hline
\end{tabular}

actividades productivas como, pequeños agricultores y ganaderos familiares, mineros artesanales de extracción de arcillas y mineros de material de arrastre.

La composición de género en los grupos etarios evidenció mayor proporción de hombres, de la cual el 91\% corresponde a edades en periodo productivo sin reporte de desempleo, dedicados a trabajar y estudiar. El 100\% de la proporción de mujeres corresponde a edades en periodo productivo, dedicadas a trabajar y estudiar, sin embargo, el 36\% de ellas se encuentran desempleadas (Figura 3).

\section{Nivel educativo}

Se encontró una mayor proporción de actores interesados con estudios de educación secundaria, de la cual el 39\% corresponde a hombres y el 35\% a mujeres. Le sigue el nivel educativo universitario representado por el 39\% de hombres y el $28 \%$ de mujeres. Luego, el nivel educativo primaria, representado por el $26 \%$ de 

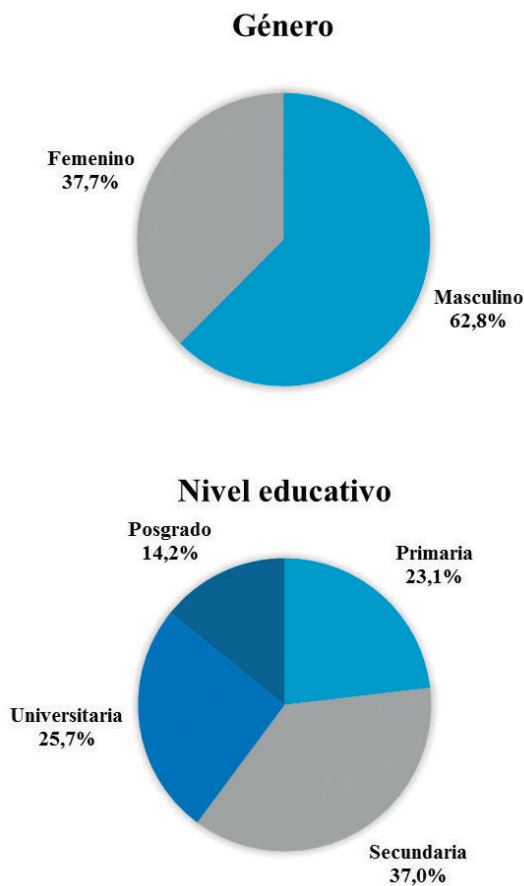
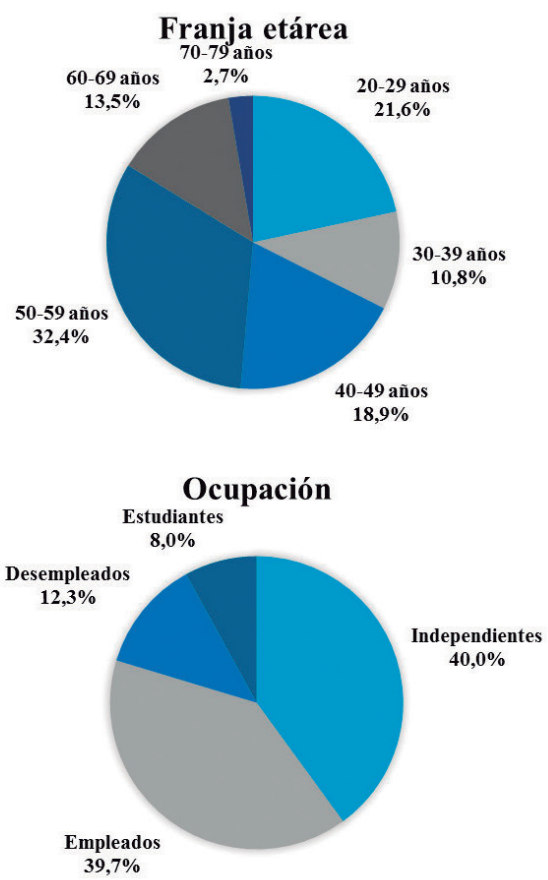

Figura 3: Composición sociodemográfica de los actores interesados.

hombres y el $21 \%$ de mujeres. Finalmente, el nivel educativo de posgrado, que se encuentra representado por el $13 \%$ de hombres y el 14\% de mujeres (Figura 3).

Para este estudio, todas las interacciones de las variables sociodemográficas no predijeron significativamente la probabilidad de identificación y valoración de ninguna categoría de servicio de la vereda Pueblillo $\left(\mathrm{X}^{2} 6, \mathrm{~N}=0,997=712, p>0.05\right)$ que se describe adelante. Los resultados sugieren que las diferencias legales, sociales, económicas y culturales no influyen en la percepción cuando se aborda una escala local de evaluación y los medios de vida de actores interesados locales son homogéneos. En contraste, estudios anteriores han encontrado que variables como la edad, el género, el nivel de educación formal, los ingresos económicos yla ubicación, son predictores de las percepciones de SE (Sodhi et al., 2010; Martin-López et al., 2012; Allendorf y Yang, 2013) y cada género, edad y la proximidad a la fuente de suministro de los SE, orienta su percepción hacia los medios de vida (Moutouama et al, 2019). Resultados similares al de este estudio encontraron Muhamad et al., (2014), para quienes la escala local favorece interacciones más directas y homogéneas entre las comunidades y el paisaje, que influyen en las percepciones de los actores interesados sobre los SE locales y no las variables sociodemográficas, por lo tanto, se sugiere que los diferentes resultados 
en los estudios tienen una estrecha relación con el contexto.

\section{Identificación de categorías de servicios ecosistémicos}

Los actores interesados identificaron indistintamente las tres categorías de servicios $\left(\mathrm{X}^{2}=712, \mathrm{p}>0.05\right)$. La categoría mayormente identificada fue la de aprovisionamiento, seguida por la de regulación y finalmente la cultural. Estudios encontrados en la literatura muestran preferencias socioculturales hacia servicios ecosistémicos de aprovisionamiento, seguido por los de regulación y, por último, los servicios culturales (Iftekhar y Takama, 2007; Agbenyega, et al., 2009; Rodríguez-
Morales et al., 2020) que sugieren la importancia de cada zona de estudio como proveedoras multifuncionales de SE. Sin embargo, el uso de diferentes metodologías muestra resultados contrastantes con los obtenidos en este estudio, con mayores porcentajes de percepción para servicios de regulación y culturales sobre los de aprovisionamiento, en los que la pertenencia territorial o la sensibilidad ante la pérdida o disminución de los servicios intangibles influencia la percepción (Piraquive 2018; Gómez y Martínez, 2018). Residentes de Pueblillo identificaron en mayor porcentaje la categoría de aprovisionamiento (51\%); las categorías de regulación $(29,8 \%)$ y cultural $(22,6 \%)$ son más identificadas por las organizaciones sociales (Figura 4).
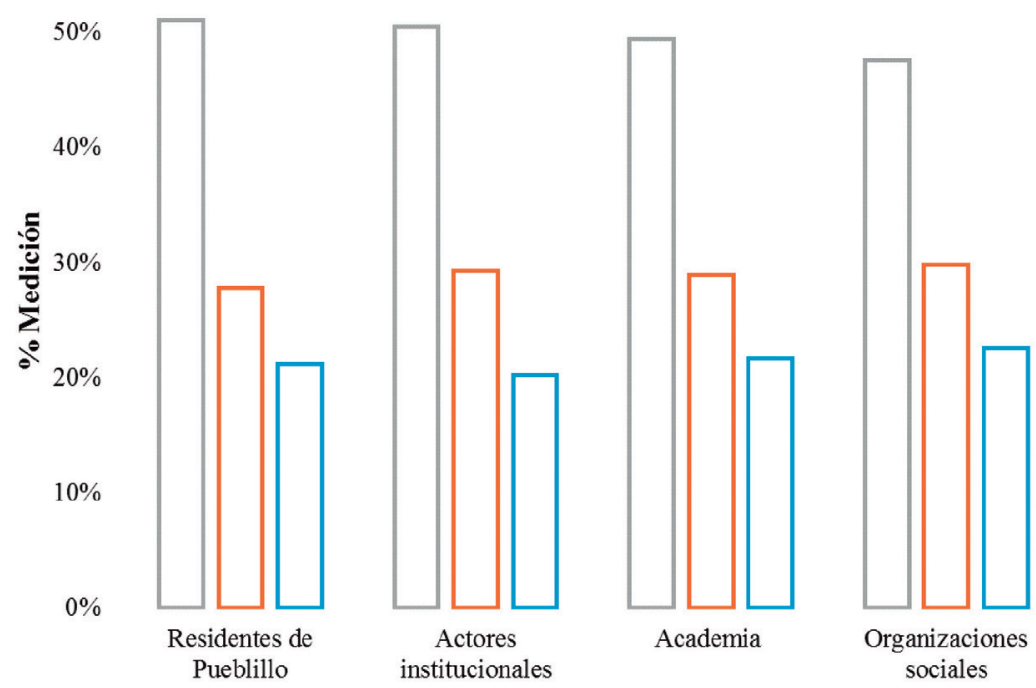

口Cultural 口Regulación $\square$ Aprovisionamiento

Actores interesados

Figura 4: Tipo de servicios ecosistémicos percibidos por los actores interesados. Actores primarios: residentes de Pueblillo y organizaciones sociales; actores secundarios: actores institucionales y academia. 
Los resultados sugieren que los actores interesados, al ubicarse en una edad promedio potencialmente productiva desde el punto de vista laboral, encuentran en la zona de estudio una fuente de servicios de aprovisionamiento que les permite suplir necesidades alimentarias y de sustento económico para acceder a mejores niveles de bienestar. Diferentes estudios de caso en todo el mundo señalan la preponderancia de los servicios de aprovisionamiento en la satisfacción de necesidades básicas y económicas (Garrido, Elbakidze y Angelstam, 2017; Moutouama et al., 2019; Koko et al., 2020). Esto se debe en gran parte a que es uno de los servicios ecosistémicos mejor estudiados (Zhang et al., 2019). Investigaciones colombianas de valoración sociocultural también muestran preferencias hacia el reconocimiento del SE de aprovisionamiento, debido a su uso más frecuente en actividades de mono y policultivos, ganadería para ordeño y madera como materia prima para la construcción (Villamagua-Vergara 2017; Gómez y Martínez, 2018).

\section{Identificación de subcategorías de SE}

En el servicio de aprovisionamiento los actores interesados identificaron quince

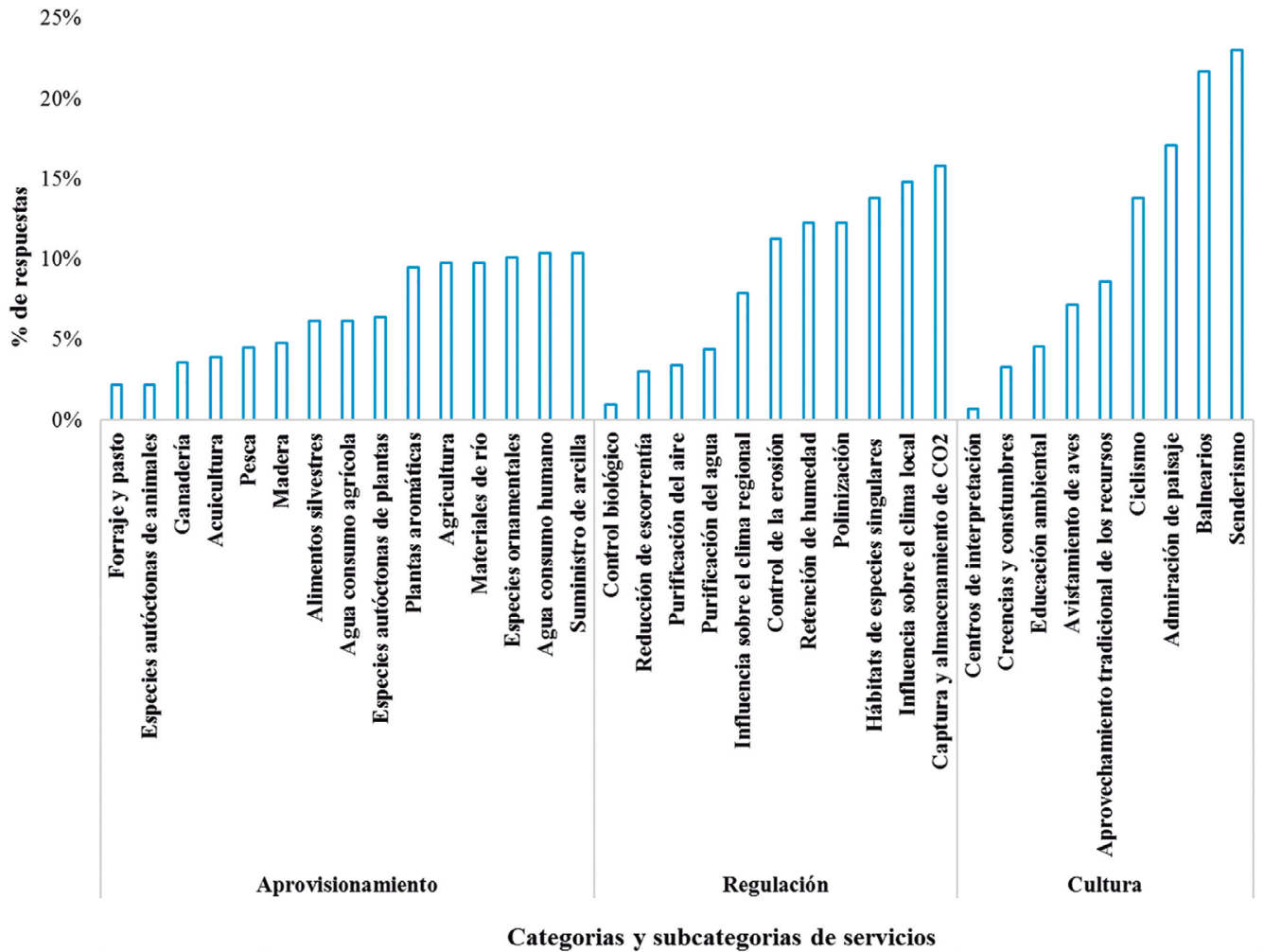

Figura 5: Sub-categorías de servicios ecosistémicos identificadas en la vereda Pueblillo. 
subcategorías, con porcentajes mayores para el suministro de arcilla (10,4\%), agua para consumo humano (10,4\%), especies ornamentales $(10,1 \%)$, material de río (9,8\%), agricultura y plantas aromáticas $(9,5 \%)$ (Figura 5).

La percepción de estas subcategorías se asocia con los modos de vida soportados históricamente en la minería artesanal de arcilla y la extracción de materiales de arrastre en el río Molino, que se intensificaron por las exigencias de desarrollo del área metropolitana y originaron en la actualidad la alteración de los valores ecológicos y la estética del paisaje de la vereda (SGG, 2015). Literatura científica en contextos similares de minería de arcilla y material de arrastre es inexistente, por lo tanto, no se realizan contrastes con otros estudios de caso. Sin embargo, se resalta que los actores interesados manifiestan la presencia de conflictos en la zona asociados con la preponderancia que tiene la propiedad del suelo y el subsuelo sobre derechos y limitaciones en su uso y apropiación. En consecuencia, los derechos de propiedad aplicados inadecuadamente son los principales impulsores de la explotación de los recursos naturales $\mathrm{y}$, actualmente, limitan la gestión de los servicios ecosistémicos (Saarikoski, 2018).

En cuanto al servicio de regulación, los actores interesados identificaron once subcategorías con porcentajes mayores para el suministro de captura y almacenamiento de $\mathrm{CO}_{2}(15,8 \%)$, influencia sobre el clima local $(14,8 \%)$, hábitats de especies singulares (13,8\%), polinización
$(12,3 \%)$, retención de humedad $(12,3 \%) \mathrm{y}$ control de la erosión (11,3\%) (Figura 5). Estas subcategorías corresponden en gran parte a procesos ecológicos intangibles cuyos beneficios son más difíciles de evidenciar y que, para su propio detrimento, pueden entrar en conflicto con los servicios de aprovisionamiento. De hecho, aunque los residentes de Pueblillo y organizaciones sociales (actores primarios), ven el territorio principalmente como fuente de sustento y los actores institucionales y la academia (actores secundarios) dirigen su percepción hacia las funciones ecosistémicas, al desagregar el servicio de aprovisionamiento, los resultados muestran que las subcategorías identificadas con porcentajes mayores, también lo son respecto a los porcentajes mayores con que se identificaron las subcategorías de aprovisionamiento. Esto sugiere que, los actores interesados perciben beneficios diferentes de acuerdo a sus roles en el territorio (Muñoz et al, 2017). Esto plantea la necesidad de estudiar los trade-offs que se presentan entre estas subcategorías de SE, con el reto de abordar la complejidad de las identidades que los actores interesados han construido, en donde subcategorías de servicios de aprovisionamiento como suministro de arcilla y materiales de río, también pueden tener la característica de intangible y la comunidad las puede consideran igual o más importantes porque hace parte de la construcción cultural del territorio (Chan, Satterfield y Goldstein, 2012).

Con respecto al servicio cultural, los actores interesados identificaron 9 
subcategorías con porcentajes mayores para senderismo (23\%), balnearios $(21,7 \%)$, admiración del paisaje (17,1\%) y ciclismo $(13,8 \%)$ (Figura 5). La inspiración estética del entorno natural de la vereda Pueblillo, se da generalmente por cuenta del mirador Broncazo y el sendero "ruta del Agua Río Molino", que permiten la visualización del paisaje y tienen potencial como atractivos turísticos, aun así, sigue siendo prioridad el desarrollo económico de la zona a partir de las actividades agrícolas y mineras. Estas subcategorías hacen parte de un área de desarrollo desconocida, ya que se las consideran intangibles o no materiales en comparación con otros servicios (Tilliger et al, 2015; Ioana et al., 2013). Sin embargo, la labor pedagógica se muestra como una herramienta útil en el conocimiento y abordaje de los SE culturales, puesto que los aprendizajes y experiencias formativas mediadas por estrategias educativas, establecen una relación de experiencia entre el ser humano y la naturaleza, además, permiten el desarrollo del sentido de pertenencia y fomentan las actividades de recreación (Dickhinson y Hobbs, 2017). En este sentido, la percepción de los servicios culturales podría aumentar considerablemente cuando la comunidad participe en programas de Educación Ambiental, puesto que les permitirá adquirir un mejor conocimiento de las áreas naturales y los beneficios que generan los ecosistemas (Casado -Arzuaga et al, 2013). Es así como los servicios culturales pueden favorecer el acceso a la naturaleza y la exposición a ecosistemas diversos y saludables (Smith et al., 2013).
Valoración de la importancia de los servicios ecosistémicos

Se encontró una relación significativa entre los servicios ecosistémicos identificados y la importancia otorgada por los actores interesados $\left(\mathrm{X}^{2}=0,000, p=0,05\right)$. El $80 \%$ ubica a los SE en dos categorías de valoración: extremadamente importante e importante. El 20\% restante de actores interesados ubica los SE en las categorías de valoración en: moderadamente importante, poco importante y nada importante. Para los residentes de Pueblillo y las organizaciones sociales, el servicio de aprovisionamiento es el más importante; para los actores institucionales y la academia, lo es el servicio de regulación, estos últimos en contraste, asignan importancia moderada y baja a la categoría de aprovisionamiento. Todos los actores interesados consideran que la categoría cultural es importante y extremadamente importante, pero con una menor proporción comparado con los SE de aprovisionamiento y regulación (Figura 6).

Los resultados sugieren que los SE con mayor importancia para los actores interesados son producto de la relación con el entorno, la cual se desarrolla en función del interés sobre él. Cuando existe alta dependencia de los SE (residentes de Pueblillo y organizaciones sociales), el interés está orientado al acceso a recursos naturales para satisfacer necesidades vitales y económicas, por lo tanto, dan mayor importancia a servicios de aprovisionamiento, mientras que para los actores interesados con alta influencia sobre los SE (actores institucionales), el 


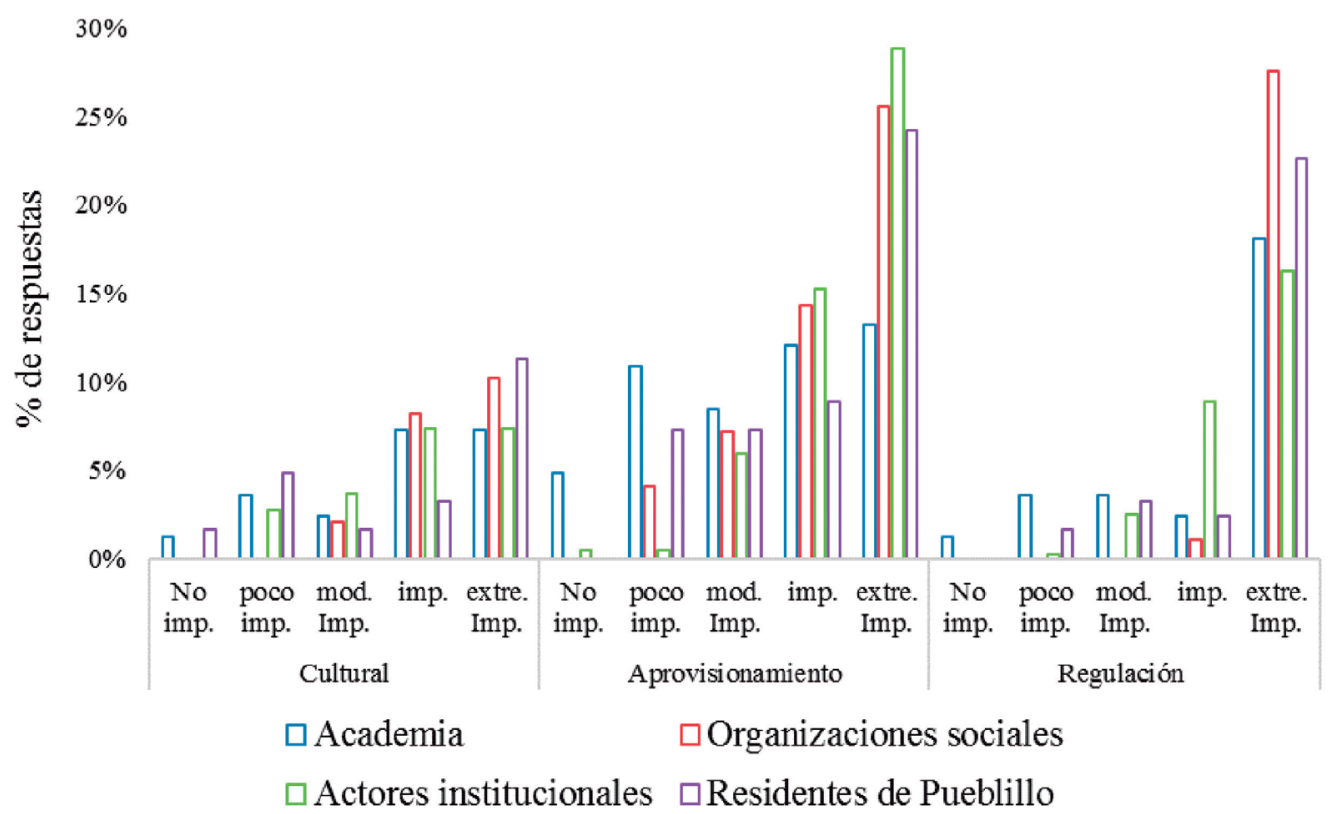

Figura 6: Importancia de los servicios ecosistémicos otorgada por los actores interesados.

interés está dirigido hacia la gestión de los recursos naturales, por ello, dan mayor importancia a servicios de regulación, que además, los ubica en una posición de mayor poder porque pueden influenciar el acceso de actores dependientes de SE.

Estudios que abordan las relaciones de poder entre actores interesados muestran que, quienes gestionan SE, determinan el acceso a servicios finales y, por lo tanto, pueden limitar su acceso para los actores menos empoderados (Felipe-Lucia et al., 2015), e.g., no todos los actores pueden acceder a la extracción de arcilla y material de arrastre, por lo cual, su percepción de importancia puede estar más orientada hacia los SE a los que sí tengan acceso. Otros estudios sobre percepción de importancia muestran que, cuando la valoración se basa en el beneficio personal, los actores interesados consideran más relevantes los servicios culturales y, cuando se basa en el beneficio social, los actores consideran más importantes los servicios de aprovisionamiento (OterosRozas et al. 2014).

La menor importancia otorgada a los servicios culturales también sugiere su menor demanda, comparada con las demás categorías de servicios. Aunque no se evidenció un sistema de conocimientos locales fuerte, los actores primarios realizan aprovechamiento tradicional de la zoca del café. Una vez el cultivo ha terminado su etapa productiva, se utiliza para la quema del ladrillo, 
actividad que hace parte de la minería artesanal y que también posee un sistema de conocimiento local. Este contexto plantea la necesidad de que los actores fortalezcan los conocimientos, usos y formas de gestionar la biodiversidad y los ecosistemas, como parte del conjunto de conocimientos locales que se mantiene de manera colectiva y son transmitidos de generación en generación, puesto que la memoria cultural desempeña un papel crucial e indispensable en la gestión de los servicios ecosistémicos (Nazarea, 2006). Los resultados también sugieren un potencial conflicto de intereses entre los actores que se dedican a la extracción de arcilla y material de arrastre y los que tienen preferencia por servicios de regulación y servicios culturales.

\section{Valoración de la importancia de subcategorías de SE}

Las subcategorías de servicios de aprovisionamiento mejor valoradas, corresponden a: agua para consumo humano, suministro de arcilla, materiales de río y agricultura (Figura 7). Los residentes de Pueblillo y las organizaciones sociales consideran que el acceso al agua para consumo humano por medio del acueducto veredal o el municipal, es determinante para el sostenimiento de la vida. Adicionalmente, el suministro de materias primas como arcilla y materiales de río, representan el principal sustento económico. Los cultivos de café, plátano, maíz, fríjol y yuca se comercializan en la comunidad local y son consumidos por las propias familias que los producen.
Si bien la agricultura y la minería tienen un rol importante en la economía local, es necesario realizar transiciones hacia sistemas que concilien la producción con los valores ecológicos y culturales de los ecosistemas, puesto que en las entrevistas cara a cara, los residentes de Pueblillo manifestaron que, debido a la extracción de arcilla, el paisaje ha tenido una transformación drástica que se evidencia en la degradación del entorno visual, en los disturbios de los cursos de agua, la disminución de la cobertura vegetal, erosión y el aumento de la vulnerabilidad frente a los movimientos en masa. Por lo tanto es recomendable, desde la planeación del territorio y toma de decisiones, generar procesos de educación ambiental y educación para la sostenibilidad, que permitan a la comunidad mejorar la comprensión sobre cómo las interacciones entre el suelo y el subsuelo determinan el control de la estructura y el funcionamiento de los ecosistemas terrestres de la zona y, estos a su vez, mejoran la integridad ecológica del territorio.

En esta misma línea, los altos porcentajes con que se identifican y valoran categoría y subcategorías del servicio de aprovisionamiento, sugieren que los actores interesados necesitan mejorar su conocimiento sobre la forma en que los sistemas de producción influencian las demás categorías de SE, tal como ha sido mencionado por diversos autores, quienes resaltan las interacciones ecológicas en el funcionamiento de los componentes del ecosistema, generando y soportando los servicios ecosistémicos de un territorio 


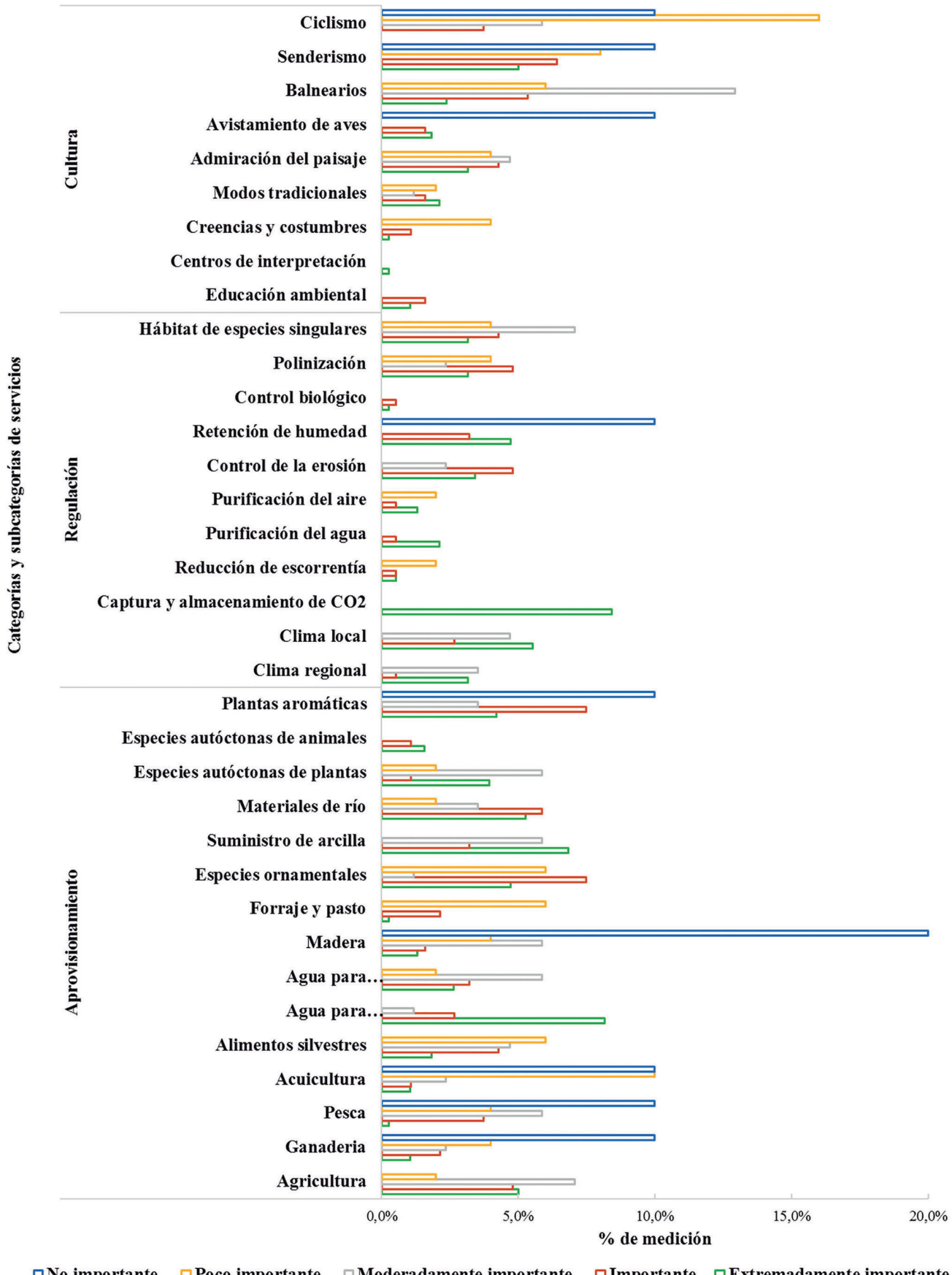

Figura 7: Importancia de las subcategorías de servicios ecosistémicos. 
para satisfacer directa o indirectamente las necesidades, usos y preferencias humanas (Daily et al., 1997; De Groot et al., 2002; Hawkins, 2003; Kremen, 2005). Adicionalmente, es necesario que comprendan cómo todas estas dinámicas se encuentran anidadas a los procesos ecológicos y sociales en escalas espaciales mayores a la local (Brondízio et al., 2010).

Las subcategorías de servicios de regulación mejor valoradas corresponden a captura y almacenamiento de dióxido de carbono y clima local. Esto se debe a la articulación entre los residentes de Pueblillo, la CRC y el Acueducto y Alcantarillado de Popayán, para cuidar un bosque en mejor estado de conservación, ubicado en la zona fragmentada que abarca las veredas El Sendero, El Hogar y Pueblillo. Los actores interesados consideran que la vegetación de esta zona mejora el clima de la vereda y captura gases contaminantes, por lo tanto, le dan mayor importancia a los SE de regulación prestados por el área en conservación, además se encuentran involucrados en su conservación y ven la función social y ecológica desde los beneficios a la salud y el bienestar de las personas y por su aportación al desarrollo social y del territorio (CRC-UICN 2015).

Las subcategorías de servicios culturales son mayormente valoradas como poco importante y no importante (Figura 7). Los actores primarios interactúan con el territorio más desde el concepto de provisión y regulación y los actores secundarios desde las categorías de regulación. La menor valoración de servicios culturales intangibles que incluyen la contemplación del paisaje y la conservación, sugieren que la zona periurbana tiene un potencial socioeconómico no aprovechado, que requiere de un proceso de acompañamiento institucional para la conformación de esta línea de aprovechamiento, que incluya la valoración económica y no solo sociocultural del territorio, a través de la innovación social que genere emprendimientos comunitarios en la línea del turismo rural y de aventura.

Estos enfoques podrían dinamizar la identidad territorial, el tejido comunitario y el aprovechamiento sostenible de los recursos naturales. Los estudios de Schmitz et al. (2003) y Castro et al. (2011), muestran que el turismo cultural y de naturaleza es de interés para las zonas rurales, además, está vinculado con el mantenimiento de prácticas de agricultura de subsistencia no intensiva, como es el caso de la agricultura que se presenta en Pueblillo. Los modelos de gestión campesina multifuncional con prácticas de bajo impacto podrían ser útiles para preservar el paisaje cultural de la zona y así potenciar el mantenimiento de una amplia gama de SE (García-Llorente et al., 2012). Otros estudios también resaltan a la agricultura tradicional como factor importante por su influencia en los valores estéticos y la identidad local (Sayadi et al., 2009).

Se considera que los aspectos físicos y tangibles del paisaje permitieron una valoración de importancia hacia los servicios ecosistémicos asociados a la recreación al aire libre, puesto que los distintos actores los experimentan 
activamente, siendo un componente esencial del entorno de las personas, una expresión de la diversidad de su patrimonio cultural y natural compartido y una base de su identidad cultural (Stephenson,2008). Este resultado es coherente con estudios previos donde los residentes perciben al menos seis tipos de servicios ecosistémicos proporcionados por su paisaje (Dou et al, 2020).

La diversidad de características del paisaje de la zona de estudio, puede haber suscitado diferentes perspectivas que contribuyen a la percepción de variados servicios culturales, por lo tanto, comprender la importancia que los actores primarios le otorgan a los servicios culturales de los ecosistemas puede ayudar a fortalecer las capacidades de gobernanza, mejorando así los medios de vida y la sostenibilidad ambiental de la zona de estudio.

\section{Conclusiones}

Con los resultados de esta investigación se acepta y afirma la hipótesis de que los diferentes actores interesados de la vereda Pueblillo comprenden y valoran desde múltiples perspectivas los servicios ecosistémicos, otorgándoles distintos grados de importancia, influenciados por factores socio-culturales de los pobladores y sus condiciones sociodemográficas.

La percepción sobre los servicios ecosistémicos de los actores primarios y secundarios presentes en la zona periurbana de Pueblillo se centra en los SE de aprovisionamiento, pues la economía local, en su mayoría, es soportada por la extracción y aprovechamiento de materias primas y el manejo agropecuario del territorio, seguido de un reconocimiento de los servicios ecosistémicos de regulación.

El menor reconocimiento y valoración de los servicios ecosistémicos de regulación tiene implicaciones en participación ciudadana y en temas de desarrollo ambiental, que se evidencian en los procesos de planificación ambiental, es por esto que la valoración sociocultural de los servicios ecosistémicos es una herramienta que apoya la toma de decisiones sobre la gestión y conservación de áreas que los proveen.

Los actores de la vereda Pueblillo actualmente no perciben el alto potencial que ofrecen los servicios ecosistémicos culturales para la dinamización de estrategias de innovación social, que aporten al desarrollo local desde el aprovechamiento cultural del territorio, por lo que se requiere implementar un proceso de valoración económica ambiental y de capacitación en modelos de gestión campesina multifuncional, turismo cultural y de aventura.

\section{Agradecimientos}

Los autores agradecen al Sistema de Investigación, Desarrollo e Innovación (SIDI) de la Fundación Universitaria de Popayán por la financiación del proyecto de investigación en la modalidad 
semilleros de investigación, convocatoria interna 047 de 2019, que dio origen a este artículo y al semillero de investigación de Gestión Social y Legislación Ambiental. De igual manera a la magister Julieth Alexandra Chacón por la ayuda brindada en la realización del mapa del área de estudio, a la comunidad de la vereda Pueblillo y a todos los actores interesados por su valioso tiempo y contribuciones al desarrollo de la investigación.

\section{Referencias}

Allendorf, T. y Yang, J. 2013. The role of ecosystem services in parkpeople relationships: The case of Gaoligongshan Nature Reserve in southwest China. Biological Conservation.167. Disponible en: https:// doi.org/10.1016/j.biocon.2013.08.013

Agbenyega, O., Burgess, P.J., Cook, M. y Morris J. 2009. Application of an ecosystem function framework to perceptions of community woodlands. Land Use Policy Vol. 26(3): 551-557. Disponible en: https:// doi.org/10.1016/j.landusepol.2008.08.011

Anheier, H. K. y Katz, H. 2004. Network approaches to global civil society. 207-221 pp. En: Anheier, H., Kaldor, M. y Glasius, M.(Ed.) Global Civil Society 2004/5. SAGE Publications, California. 392Pp.

Aryal, K., Ojha, B. y Maraseni, T. 2021. Perceived importance and economic valuation of ecosystem services in Ghodaghodi wetland of Nepal. Land Use Policy. 106: 105450. Disponible en: https://doi. org/10.1016/j.landusepol.2021.105450

Balvanera, P., Castillo, A. y MartÍnez-Harms, M.J. 2011. Ecosystem Services in Seasonally Dry Tropical Forests. In: Dirzo R., Young H.S., Mooney H.A., Ceballos G. (eds) Seasonally Dry Tropical Forests. Island Press, Washington, DC. Disponible en: https://doi. org $/ 10.5822 / 978-1-61091-021-7 \_15$ 
Balvanera, P., Siddique, I., Dee, L.A., Paquette, A., Isbell, F.I., Gonzalez, A., Byrnes, J., O’Connor, M.I., Hungate, B.A. y Griffin, J. 2014. Linking Biodiversity and Ecosystem Services: Current Uncertainties and the Necessary Next Steps. BioScience. 64(1): 49-57. Disponible en: https://doi. org/10.1093/biosci/bitoo3

Balvanera, P., Castillo, A., Lazos, E., Caballero, K., Quijas, S., Flores, A., Galicia, C., Martínez, L., Saldaña, A., Sanchez, M., Maass, M., Avila, P., Martínez, Y., Galindo, L.M. y Sarukhán, J. 2013. Marcos Conceptuales Interdisciplinarios Para El Estudio De Los Servicios Ecosistémicos En América Latina.Pp 38-67. En: Laterra,P., Jobbágy, E. y Paruelo,J.(Ed) Valoración de Servicios Ecosistémicos. Conceptos, Herramientas y Aplicaciones para el Ordenamiento Territorial. INTA. Buenos Aires Argentina.726 pp.

Brondízio, E.S., Gatzweiler, F., Kumar, M. y Zograftos, C. 2010. Socio-cultural context of ecosystem and biodiversity valuation. Pp 149-174.En, Pushpam, K.(Ed) The Economics of Ecosystems and Biodiversity Ecological and Economic Foundations. 456pp.

Bullock, C., Joyce, D. y Collier M., 2018. An exploration of the relationships between cultural ecosystem services, sociocultural values and well-being. Ecosystem Services, 31, (A): 142152. Disponible en: https://doi.org/10.1016/j.ecoser.2018.02.020

Casado -Arzuaga, I., Madariaga, I., Onaindia, M., y Verburg, P. H. 2013. Perception, demand and user contribution to ecosystem services in the Bilbao Metropolitan Greenbelt. Journal of Environmental Management. 129. 33-43. Disponible en: https://doi.org/10.1016/j.jenvman.2013.05.059

Castro, A.J., Martín-López, B., García-LLorente, M., Aguilera, P. A., López, E., y Cabello, J. 2011. Social preferences regarding the delivery of ecosystem services in a semiarid Mediterranean region. Journal of Arid Environments, 75(11), 1201-1208. Disponible en: https://doi.org/10.1016/j.jaridenv.2011.05.013 
CEPAL, NU. 2008. Panorama Social de América Latina. CEPAL. 258 pp.

Chan, K., Guerry, A.D., Balvanera, P., Klain, S., Satterfield, T., Basurto, X., Bostrom, A., Chuenpagdee, R., Gould, R., Halpern, B.S., Hannahs, N., Levine, J., Norton, B., Ruckelshaus, M., Russell, R.,Tam,.J. y Woodside, U. 2012. Where are 'Cultural' and Social in Ecosystem Services? A Framework for Constructive Engagement. BioScience. 62(8): 744-756. Disponible en: https:// doi.org/62.744-756.10.1525/bio.2012.62.8.7

Comisión europea, 2017. Openness operationalisation of natural capital and ecosystem services: from concepts to realworld applications. Disponible en: https://cordis.europa.eu/ project/id/308428/reporting

Corporación Autónoma Regional del Cauca CRG y Unión Internacional Para La Conservación de la Naturaleza UICN. 2015. Diagnóstico, Identificación y Valoración económica de Servicios Ecosistémicos, municipios de San Juan Nepomuceno y Santa Rosa de Cauca, objetivo 6. Disponible en: https:// www.iucn.org/sites/dev/files/content/documents/obj_6_ valoracion_santa_rosa_de_cauca.pdf

Cresswell JW, Plano Clark VL. 2011.Designing and conducting mixed method research. 2nd Sage; Thousand Oaks, CA.

Daily G.C., Alexander S, Ehrlich P.R., Goulder L., Lubchenco J., Matson P.A., Mooney, H.A., Postel, S., Schneider, S.H., Tilman, D. y Woodwell, G.M. 1997. Ecosystem services: benefits supplied to human societies by natural ecosystems. Issues in Ecology 2:1-18. Disponible en: https://www.esa.org/wp-content/ uploads/2013/o3/issue2.pdf

Dickinson D.C. y Hobbs R.J. 2017. Cultural ecosystem services: Characteristics, challenges and lessons for urban green space research, Ecosystem Services, 25: 179-194. Disponible en: https://doi.org/10.1016/j.ecoser.2017.04.014Dou, Y., Yu, X., Bakker, M.M., Groot, R., Carsjens, G., Houlang, D. y Huang, C. 2020. Analysis of the relationship between cross- 
cultural perceptions of landscapes and cultural ecosystem services in Genheyuan region, Northeast China. Ecosystem Services. 43: 101112. Disponible en: https://doi.org/10.1016/j. ecoser.2020.101112

Durham E., Baker H., Smith M., Moore E. y Morgan V. 2014. The BiodivERsA Stakeholder Engagement Handbook. BiodivERsA, Paris. 108 pp.

Felipe-Lucia, M. R., Martín-López, B., Lavorel, S., Berraquero- Díaz, L., Escalera-Reyes, J. y Comín, F.A. 2015. Ecosystem services flows: why stakeholders' power relationships matter. PLoS ONE 10(7): e0132232. Disponible en: https://doi.org/10.1371/journal.pone.0132232García-Nieto, A. P., Quintas-Soriano, C., García-Llorente, M., Palomo, I., Montes, C., y Martín-López, B. 2014. Collaborative mapping of ecosystem services: The role of stakeholders' profiles. Ecosystem Services. 13: 141-152. Disponible en: https://doi. org/10.1016/j.ecoser.2014.11.0o6

Garrido, P., Elbakidze, M., y Angelstam, P. 2017. Stakeholders' perceptions on ecosystem services in Östergötland's (Sweden) threatened oak wood-pasture landscapes. Landscape and Urban Planning. 158. 96-104. Disponible en: https://doi.org/10.1016/j.landurbplan.2016.08.018.

Gómez, F.C.A. 2012. Análisis territorial de las veredas periurbanas de Popayán ante la presión urbana, estudio de caso, vereda Crucero Puelenje. Trabajo de grado Maestría. Universidad Autónoma de Manizales. Facultad de estudios Sociales y Empresariales. Popayán-Cauca. 287pp.

Gómez, W.S. y Martinez, N.D. 2018. Evaluación de la percepción sociocultural de los servicios ecosistémicos en la cuenca del río Orotoy aplicando la metodología de proceso analítico jerárquico-ahp. Universidad Santo Tomás. Facultad de Ingeniería Ambiental, trabajo de grado en Ingeniero Ambiental. Villavicencio-Meta.84 pp. 
Ghai D, Vivian J.M. 2014. Grassroots environmental action: people's participation in sustainable development. Routledge, New York, USA. 374 pp.

De Groot, R., Wilson, A. M., Boumans, M. J. 2002. A typology for the classification, description and valuation of ecosystem functions, goods and services. Ecological Economics 41. Pp 393-498. Disponible en: https://doi.org/10.1016/So9218009(02)ooo89-7 De Groot, R., Stuip, M., Finlayson, M. y Davidson, N. 2006. Valoración de humedales: Lineamientos para valorar los beneficios derivados de los servicios de los ecosistemas de humedales CBD Technical Series, 27. Fecha de acceso: 2021 agosto 3.

De Groot, R., Fisher, B., Christie, M., Aronson, J., Braat, L., Gowdy, J., Haines-Young, R., Maltby, E., Neuville, A., Polasky, S., Portela, P y Ring, I. 2010. Integrating the ecological and economic dimensions in biodiversity and ecosystem service valuation. Pp 9-40. En Kumar, P. (Ed.). The Economics of Ecosystems and Biodiversity: Ecological and Economic Foundations, Earthscan Routledge, Londres. 422 pp.

Hauck, J., Saarikoski, H., Turkelboom, F. y Keune, H. 2016. Stakeholder Analysis in ecosystem service decision-making and research. In: Potschin, M. and K. Jax (eds): OpenNESS Ecosystem Services Reference Book. EC FP7 Grant Agreement no. 308428. Disponible en: www.openness-project.eu/library/ reference-book

Koko I.A., Misana S.B., Kessler A y Fleskens L. 2020. Valuing ecosystem services: stakeholders' perceptions and monetary values of ecosystem services in the Kilombero wetland of Tanzania, Ecosystems and People, 16:1, 411-426, Disponible en: https://doi.org/10.1080/26395916.2020.1847198

Hawkins K. 2003. Economic valuation of ecosystem services. University of Minnesota, 23. Disponible en:http://citeseerx.ist.psu. edu/viewdoc/download?doi=10.1.1.473.4949\&rep=rep1\&type=pdf 
IBM Corp. Released 2017. IBM SPSS Statistics for Windows, Version 25.0. Armonk, NY: IBM Corp.

Iftekhar, S. y Takama, T. 2007. Perceptions of biodiversity, environmental services, and conservation of planted mangroves: A case study on Nijhum Dwip Island, Bangladesh. Wetlands Ecology and Management. 16. 119-137. Disponible en: https://doi.org/10.1007/S11273-007-9060-8

Iniesta-Arandia, I., Garcia-Llorente, M., Aguilera, P.A., Montes, C. y Martín-López, B. 2014. Socio-cultural valuation of ecosystem services: Uncovering the links between values, drivers of change, and human well-being. Ecological Economics. 108: 36-48. Disponible en: https://doi. org/10.1016/j.ecolecon.2014.09.028

Iniguez-Gallardo, V., Halasa, Z. and Briceño, J. 2018. People's Perceptions of Ecosystem Services Provided by Tropical Dry Forests: A Comparative Case Study in Southern Ecuador. IntechOpen.78. 96-112. Disponible en: https://doi.org/10.5772/ intechopen.75081

Instituto de Investigación de Recursos Biológicos Alexander von Humboldt [Internet]. 2014. Valoración Integral de la Biodiversidad y los Servicios Ecosistémicos (VIBSE), aspectos conceptuales y metodológicos. Disponible en: http://hdl. handle.net/20.500.11761/32547

Milcu, A., Ioana, J., Hanspach, D., Abson, y Fischer, J. 2013. Cultural ecosystem services: a literature review and prospects for future research. Ecology and Society 18(3):44. Disponible en: http://dx.doi.org/10.5751/ES-05790-180344

Kandel, P., Tshering, D., Uddin, K., Lhamtshok, T., Aryal, K., Karki, S., Sharma, B. y Chettri, N. 2018. Understanding social-ecological interdependence using ecosystem services perspective in Bhutan, Eastern Himalayas. Ecosphere 9(2): e02121. Disponible en: https://doi.org/10.1002/ecs2.2121 
Kremen, C. 2005. Managing ecosystem services: what do we need to know about their ecology? Ecology Letters. 2005; 8:468-479. Disponible en: https://doi.org/10.1111/j.14610248.2005.00751.X

Olander L., Polasky S., Kagan J.S., Johnston R.J., Wainger L., Saah D.,Maguire L., Boyd J. y Yoskowitz D. 2017. So you want your research to be relevant? Building the bridge between ecosystem services research and practice, Ecosystem Services, Volume 26, Part A, Pages 170-182, ISSN 2212-0416, https://doi. org/10.1016/j.ecoser.2017.06.003.

Malinauskaite, L., Davidsdottir, B. y Ögmundardottir, H. 2020. Socio-cultural valuation of whale ecosystem services in Skjálfandi Bay, Iceland. Ecological Economics. 180: 106867. Disponible en: https://doi.org/10.1016/j.ecolecon.2020.106867

Maguire, B., Potts, J. y Fletcher, S. 2012. The role of stakeholders in the marine planning process-Stakeholder analysis within the Solent, United Kingdom. Marine Policy, Volume 36: 246257. Disponible en: https://doi.org/10.1016/j.marpol.2011.05.012

Martín-López, B. y Montes, C. 2011. Biodiversidad y servicios de los ecosistemas. Biodiversidad en España: base de la sostenibilidad ante el cambio global. Observatorio de la Sostenibilidad en España. 444-465.

Martín-López, B., Iniesta-Arandia, I., Garcia-Llorente, M., Palomo, I., Casado Arzuaga, I., Amo, D.G.D., Gómez Baggethun, E., Oteros-Rozas, E., Palacios Agundez, I., Willaarts B., González, J., Santos-Martin, F., Onaindia, M., López Santiago, C. y Montes, C. 2012. Uncovering Ecosystem Service Bundles through Social Preferences. PloS one. 7. e38970. Disponible en: https://doi.org/10.1371/journal.pone.0038970

Maynard, S., James, D. y Davidson, A. 2010. The Development of an Ecosystem Services Framework for South East Queensland. Environmental management, 45:881-95. Disponible en: https:// doi.org/10.1007/SO0267-010-9428-Z 
Mensah S., Veldtman R., Assogbadjo A E., Ham C., Glèlè Kakaï R. y Seifert T. 2017. Ecosystem service importance and use vary with socio-environmental factors: A study from household-surveys in local communities of South Africa, Ecosystem Services, 23:1-8, ISSN 2212-0416. Disponinble en: https://doi.org/10.1016/j.ecoser.2016.10.018

Moutouama, F., Biaou, S.S., Kyereh, B., Asante, W., y Natta, A. 2019. Factors shaping local people's perception of ecosystem services in the Atacora Chain of Mountains, a biodiversity hotspot in northern Benin. Journal of Ethnobiology and Ethnomedicine. 15(38). Disponible en: https://doi.org/10.1186/ S13002-019-0317-0

Muhamad, D., Okubo, S., Harashina, K., Parikesit., Gunawan, P. and Takeuchi, K. 2014. Living close to forests enhances people's perception of ecosystem services in a forestagricultural landscape of West Java, Indonesia. Ecosystem Services. 8: 197-206. Disponible en: https://doi.org/10.1016/j. ecoser.2014.04.003

Nazarea, V. 2006. Local Knowledge and Memory in Biodiversity Conservation. Annual Review of Anthropology. 35:317-335. Disponible en: https://doi.org/10.1146/annurev. anthro.35.081705.123252

Nieto-Romero, M., Oteros-Rozas, E., González, J.A. y Martín-López, B. 2014. Exploring the knowledge landscape of ecosystem services assessments in Mediterranean agroecosystems: insights for future research. Environmental Science \& Policy 37:121-133. Disponible en: http://dx.doi. org/10.1016/j.envsci.2013.09.003.

Notte, A., D’Amato, D., Mäkinen, H., Paracchini, M.L., \& Liquete, C., Egoh, B.N., Geneletti, D. and Crossman, N. 2013. Original Articles Ecosystem services classification: A systems ecology perspective of the cascade framework. Ecological Indicators. 74: 392-402.Disponible en: https://doi. org/10.1016/j.ecolind.2016.11.030 
Oteros-Rozas, E., Martín-López, B., González,J.A., Plieninger, T., López, C. y Montes, C. 2014. Socio-cultural valuation of ecosystem services in a transhumance social-ecological network. Reg Environ Change 14:1269-1289. https://doi. org/10.1007/S10113-013-0571-y

Piraquive, R.C y Velasquez, M.S. 2018. Valoración integral de los servicios ecosistémicos del humedal brisas del llano en el municipio de Restrepo. Universidad Santo Tomás. Facultad de ingeniería ambiental trabajo de grado en ingeniero ambiental. Villavicencio-Meta. 79 pp.

Primmer, P., Jokinen, M., Blicharska, D.N., Barton, R., Bugter, M. y Potschin. 2015. A framework for empirical analysis of ecosystem services governance Ecosyst. Serv., pp.158-166.

Quijas, S., Schmid, B. y Balvanera, P. 2010. Plant diversity enhances provision of ecosystem services: a new synthesis. Basic and Applied Ecology 11: 582-593. Disponible en: https:// doi.org/10.1016/j.baae.2010.06.009

Ramírez -Chávez, H, E., Pérez y Ramírez, M,J. 2008.Mamiferos presentes en el municipio de Popayán, Cauca- Colombia. Boletín Científico Centro de Museos. Museo de Historia Natural, 12:65-89. Disponible en: http://www.scielo.org.co/pdf/ $\mathrm{bccm} / \mathrm{v12n1} / \mathrm{v} 12 \mathrm{n} 1 \mathrm{a0} 5 . \mathrm{pdf}$

Reed, M.S., Graves, A., Dandy, N., Posthumus, H., Hubace, K., Morris, J., Prell, C., Quinn, C.H y Stringer, L.C. 2009. Who's in and why? A typology of stakeholder analysis methods for natural resource management. Journal of Environmental Management. 90:1993-1949. Disponible en: https://doi. org/10.1016/j.jenvman.2009.01.001

Reed, M.S., Vella, S., Challies, E., de Vente, J., Frewer, L., Hohenwallner-Ries, D., Huber, T., Neumann, R.K., Oughton, E.A., Sidoli del Ceno, J. and van Delden, H. 2018. A theory of participation: what makes stakeholder and public engagement in environmental management work? Restor Ecol, 26: S7-S17. Disponible en: https://doi.org/10.1111/rec.12541 
Rodríguez-Morales, Beatriz., Roces-Díaz, José V., Kelemen, Eszter., Pataki, György., Díaz-Varela, Emilio. 2020. Perception of ecosystem services and disservices on a peri-urban communal forest: Are landowners' and visitors' perspectives dissimilar? Ecosystem Services, 43: 101089, Disponible en: https://doi.org/10.1016/j.ecoser.2020.101089.

Saarikoski H., Primmer E., Saarela SR, Antunes P., Aszalós R, Baró F., Berry P., Garcia GB., Goméz-Baggethun E., Carvalho L., Dick J., Dunford R., Hanzu M., Harrison PA., Izakovicova Z., Kertész M., Kopperoinen L., Köhler B., Langemeyer J., Lapola D., Liquete C., Luque S., Mederly P., NiemeläJ., Palomo I., Martinez Pastur G., Peri PL., Preda E., Priess JA., Santos R., Schleyer C., Turkelboom F., Vadineanu A., Verheyden W., Vikström S., Young J. 2018. Institutional challenges in putting ecosystem service knowledge in practice, Ecosystem Services, 29(Part C): 579-598, ISSN 2212-0416. Disponible en: https://doi.org/10.1016/j.ecoser.2017.07.019

Sayadi, S., González-Roa, M.C. y Calatrava-Requena, J., 2009. Public preferences for landscape features: the case of agricultural landscape in mountainous Mediterranean areas. Land Use Policy 26(2): 334e344. Disponible en: https:// doi.org/10.1016/j.landusepol.2008.04.003

Servicio Geológico Colombiano SGG. 2015. Zonificación geo mecánica y de amenaza por movimientos en masa del municipio de Popayán -Cauca. Fecha de acceso: 2021 agosto 3. Disponible en: http://recordcenter.sgc.gov.co/ B7/21003002524656/Documento/Pdf/210524656110100o.pdf

Schmitz, M.F., De Aranzabal, I., Aguilera, P., Rescia, A.J. y Pineda, F.D. 2003. Relationship between landscape typology and socioeconomic structure: scenarios of change in Spanish cultural landscapes. Ecological Modelling 168(3): 343-356. Disponible en: https://doi.org/10.1016/S0304-380o(03)00145-5 
Sodhi, N.S., Lee, T.M., Sekercioglu, C.H., Webb, E.L., Prawiradilaga, D.M., Lohman, D.J, Pierce, N., Diesmos, A.C., Rao, M. y Ehrlich, P.R. 2010. Local people value environmental services provided by forested parks. Biodiversity and Conservation. 19: 1175-1188. Disponible en: https://doi. org/10.1007/S10531-009-9745-9

Smith, L.M., Case, J. L., Smith, H. M., Harwell, L. C. y Summers, J.K. 2013. Relating ecosystem services to domains of human well-being: Foundation for a U.S. index. Ecological Indicators. 28: 79-90. Disponible en: https://doi.org/10.1016/j. ecolind.2012.02.032Stephenson, J. 2008. The Cultural Values Model: An Integrated Approach to Values in Landscapes. Landscape and Urban Planning. 84(2): 127-139. Disponible en: https://doi.org/10.1016/j.landurbplan.2007.07.003

Tengö, M., Brondízio, E., Elmqvist, T., Malmer, P. y Spierenburg, M. 2014. Connecting Diverse Knowledge Systems for Enhanced Ecosystem Governance: The Multiple Evidence Base Approach. AMBIO A Journal of the Human Environment. In press. Disponible en: https:/doi.org/10.1007/s13280-014-0501-3

Tilliger, B., Rodríguez-Labajos, B., Bustamante, J. y Settele, J. 2015. Disentangling Values in the Interrelations between Cultural Ecosystem Services and Landscape Conservation-A Case Study of the Ifugao Rice Terraces in the Philippines. Land. 4. 888-913. Disponible en: https://doi.org/10.3390/land4030888

Turner, W. R., K. Brandon, T. M. Brooks, C. Gascon, H. K. Gibbs, K. S. Lawrence, R. A. Mittermeier y E. R. Selig. 2012. Global biodiversity conservation and the alleviation of poverty. BioScience 62:85-92. Disponible en: https://doi.org/10.1525/ bio.2012.62.1.13

Vallet, A., Locatelli, B., Levrel, H., Dendoncker, N., Barnaud, C. y Quispe Conde, Y. 2019. Linking equity, power, and stakeholders' roles in relation to ecosystem services. Ecology and Society 24(2):14. Disponible en: https://doi.org/10.5751/ES-10904-240214 
De Vente J., Reed M. S., Stringer, L. C., Valente, S. y Newig J. 2016. How does the context and design of participatory decision-making processes affect their outcomes? Evidence from sustainable land management in global drylands. Ecology and Society 21:24. Disponible en: http://dx.doi. org/10.5751/ES-08053-210224

Vihervaara, P., Rönkä, M. y Walls, M. 2010. Trends in Ecosystem Service Research: Early Steps and Current Drivers. Ambio. 39(4): 314-324. Disponible en: http://dx.doi. org/10.1007/S13280-010-0048-X

Vilardy Quiroga, S., González, J.A., Martín-López, B. y Oteros-Rozas, E. 2012.Los servicios de los ecosistemas de la Reserva de Biosfera Ciénaga Grande de Santa Marta. Revista Iberoamericana de Economía Ecológica. 19: 6683. Disponible en: https://dialnet.unirioja.es/servlet/ articulo? codigo $=5007227$

Villamagua-Vergara, G. C. 2017. Percepción social de los servicios ecosistémicos en la microcuenca El Padmi, Ecuador. Revista iberoamericana de economía ecológica.27: 102-114. Disponible en: https://ddd.uab.cat/pub/revibec/ revibec_a2017v27/revibec_a2017v27p102.pdf

Yameogo, V. 2017. Ecosystem services management: An analysis of stakeholders' perceptions in Thiou, Yatenga Province, Burkina Faso. ILRI Project Report.Nairobi, Kenya: International Livestock Research Institute (ILRI).

Zhang, X., Estoque, R., Hualin, X., Murayama, Y., y Ranagalage, M. 2019. Bibliometric analysis of highly cited articles on ecosystem services. PLOS ONE. 14. e0210707. Disponible en: http://dx.doi.org/10.1371/journal.pone.0210707

Zograftos, G. y Kumar, M. 2010. Socio-cultural context of ecosystem and biodiversity valuation. Pp.149-174. En: Pushpam, K. (Ed) The Economics of Ecosystems and Biodiversity: The Ecological and Economic Foundations: Earthscan Routledge. Londres. 422pp. 